\title{
Myelopathy-mimicking symptoms of epidural venous engorgement and syringomyelia due to inferior vena cava stenosis at the thoracolumbar junction in a patient with Budd-Chiari syndrome
}

\author{
Jung-Hee Lee, MD, Wook-Jae Song, MD, and Kyung-Chung Kang, MD \\ Department of Orthopedic Surgery, Kyung Hee Medical Center, Kyung Hee University School of Medicine, Seoul, \\ Republic of Korea
}

\begin{abstract}
Epidural venous engorgement can result from various lesions, such as arteriovenous malformation, thrombosis or occlusion of the inferior vena cava (IVC), or an abdominal masslike lesion. Most patients with these problems complain of low-back pain, radicular pain, or neurogenic claudication, which are symptoms suggestive of disc herniation or spinal stenosis. However, these patients rarely exhibit neurological deficits or cauda equina syndrome. The authors encountered a case of a 60-year-old man presenting with lower-extremity weakness and voiding difficulty for a period of 1 year. To investigate the patient's myelopathy-mimicking symptoms, a lumbar spine MRI scan was performed. The MR images exhibited tortuous and dilated spinal vessels compressing the spinal cord and thecal sac at the T11-L3 level, which were concurrent with syringomyelia evidenced by a $22 \times 2.5-\mathrm{mm}$ cyst at the T11-12 level. 3D CT scanning of the whole aorta revealed total occlusion and regression of the IVC in the intrahepatic region $3 \mathrm{~cm}$ inferior to the right atrium and dilation of multiple collateral veins. The patient was diagnosed with chronic Budd-Chiari syndrome Type I. The authors performed venography, followed by intrahepatic IVC recanalization via stent placement under fluoroscopic and ultrasonographic guidance and without surgical exploration. After this treatment, there was a marked decrease in epidural venous engorgement and the patient's symptoms resolved almost completely. This case indicates that epidural venous engorgement at thoracolumbar levels may cause symptoms suggestive of myelopathy and can be successfully treated by minimally invasive procedures to eliminate the underlying causes.
\end{abstract}

http://thejns.org/doi/abs/10.3171/2015.1.SPINE14515

KEY WORDS epidural venous engorgement; syringomyelia; Budd-Chiari syndrome; myelopathy; thoracic

$\mathrm{E}$ PIDURAL venous engorgement can result from various lesions, such as arteriovenous malformation, ${ }^{4}$ thrombosis or occlusion of the inferior vena cava (IVC), ${ }^{10}$ or an abdominal masslike lesion. ${ }^{2}$ Patients with these problems commonly complain of symptoms suggestive of lumbar disc herniation or spinal stenosis. ${ }^{10}$ Most of these patients present with low-back pain, radicular pain, or neurogenic claudication, but they rarely exhibit serious symptoms such as neurological deficit or cauda equina syndrome. ${ }^{8}$
Lower-extremity weakness and voiding difficulty are generally considered to indicate serious complications due to thoracolumbar spinal diseases. The majority of patients with these symptoms are primarily considered candidates for spinal surgical procedures.

We encountered a case of Budd-Chiari syndrome in a 60-year-old male patient who presented with lower-extremity weakness and voiding difficulty due to epidural venous engorgement and syringomyelia at the thoracolumbar level, which were both caused by inferior vena cava 
stenosis. We successfully treated this patient with radiological intervention without surgical exploration. Here, we report our case with a review of the relevant literature.

\section{Case Report}

A 60-year-old man visited the outpatient clinic of our medical institution with the primary complaint of a 1-year history of weakness of both lower extremities and voiding difficulty. The patient also complained of mild-to-moderate pain in the back and both extremities. The patient had been treated for these symptoms at the department of neurology of another university hospital, but no improvement was achieved. Prior to referral to us, the patient experienced progressive weakness and numbness in both lower extremities, accompanied by voiding difficulty. The patient also had gait disturbance. On physical examination, the patient had remarkable lower-extremity weakness, which was notable in the ankle and big toe (motor power grade $[0=$ total paralysis, $5=$ active movement against full resistance, right/left]: hip flexion $5 / 5$, knee extension 4/4, ankle dorsiflexion $4 / 3$, ankle plantar flexion $4 / 3$, big toe dorsiflexion $4 / 1$, and big toe plantar flexion $4 / 3$; deep tendon reflex: knee jerk $+/+$ and ankle jerk $+/+$ ). We performed lumbar spine MRI to confirm the possible presence of spinal stenosis or intervertebral disc herniation.

Lumbar spine MR images demonstrated tortuous and dilated spinal vessels compressing the spinal cord and thecal sac at the T11-L3 level, concurrent with syringomyelia evidenced by a $22 \times 2.5-\mathrm{mm}$ cyst at the T11-12 level (Fig. 1). On subsequent thoracic spine MRI scans with wholespine sagittal views, dilation of the azygos and hemiazygos veins as well as paravertebral vessels was visible and was accompanied by the presence of a $17 \times 3-\mathrm{mm}$ cyst at the T10-11 level. After confirming the abnormal venous structures, we performed 3D CT scanning of the whole aorta. This revealed total occlusion and regression of the IVC in the intrahepatic region $3 \mathrm{~cm}$ inferior to the right atrium. Both hepatic veins drained to the remaining IVC just at the low portion of the right atrium, and a small portion of the left hepatic vein was connected to the left pericardiophrenic vein, which was severely dilated and drained to the brachiocephalic vein. The patient also had dilation of multiple collateral veins, accompanied by severe lesions suggestive of varices in the azygos, hemiazygos, splenic, gastric, and paravertebral veins. There were no seriously distended or engorged paraumbilical veins. On the basis of these findings, we diagnosed chronic Budd-Chiari syndrome Type I in this patient.

After consultation with the department of radiological intervention at our institution, we performed intrahepatic IVC recanalization using a stent. To perform IVC recanalization, we first used venography to detect the presence of a 3-cm total occlusion of the IVC. Under fluoroscopic and ultrasonographic guidance, the right internal jugular and femoral veins were punctured. Pigtail catheters were inserted at both sides, and an IVC venogram was performed. The venogram confirmed a $3-\mathrm{cm}-$ long total occlusion and aberrant multiple collateral veins at the occluded end. We considered it difficult to connect both ends of the occluded portion from the internal jugular and femoral veins directly; therefore, to evaluate the vessels adjacent to the IVC, a biplanar venogram was obtained using the pigtail at the side of the femoral vein. The operator confirmed that the collateral vessel at the left and anterior side of the hepatic segment IVC was closest to the superior portion of the hepatic segment IVC. We performed a puncture using a 16-gauge Colapinto needle and inserted a $12-\mathrm{mm} / 8-\mathrm{cm}$ stent graft (S \& G Biotech) after preballooning with an IN.PACT Admiral drug-eluting balloon (Medtronic). On subsequent tachography, the patient showed an insufficient stent expansion and poor perfusion through the stent. We therefore performed postballooning using a Conquest guidewire and an IN.PACT Admiral drug-eluting balloon. Tachography then showed a shift of perfusion to the stent graft, accompanied by a decrease of perfusion via the collateral vessels (Fig. 2).

At the 1-month follow-up, the patient's thoracolumbar MRI scans showed a marked decrease in epidural venous engorgement. However, persistent syringomyelia was evident at the lower thoracic levels (Fig. 3). Whole-aorta CT
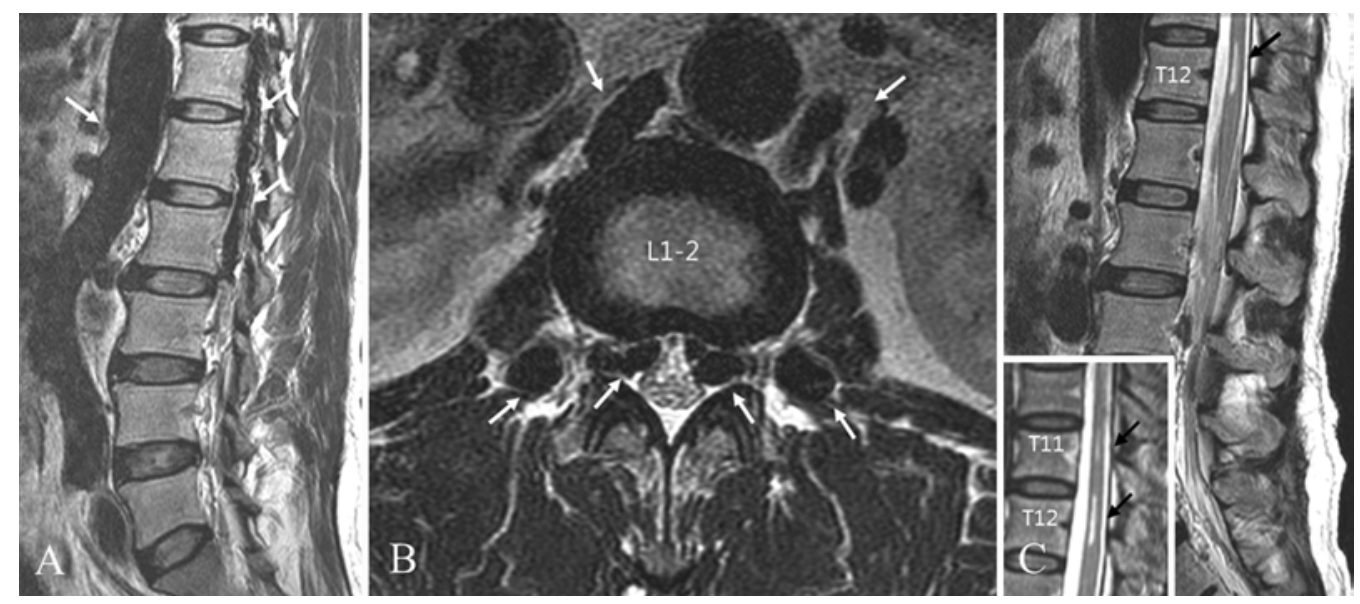

FIG. 1. A: Initial lumbar sagittal T2-weighted MR image showed multiple engorgements of the hemiazygos vein and prevertebral vessels with multiple intraforaminal extensions at T11-L3 (white arrows). B: Axial T2-weighted MRI scan showed multiple epidural vein engorgements with compression of the dural sac and neural foramen at the conus medullaris level (white arrows). C: In addition, there were $17 \times 3-\mathrm{mm}$ and $22 \times 2.5-\mathrm{mm}$ cysts at T10-11 and T11-12 (black arrows). 

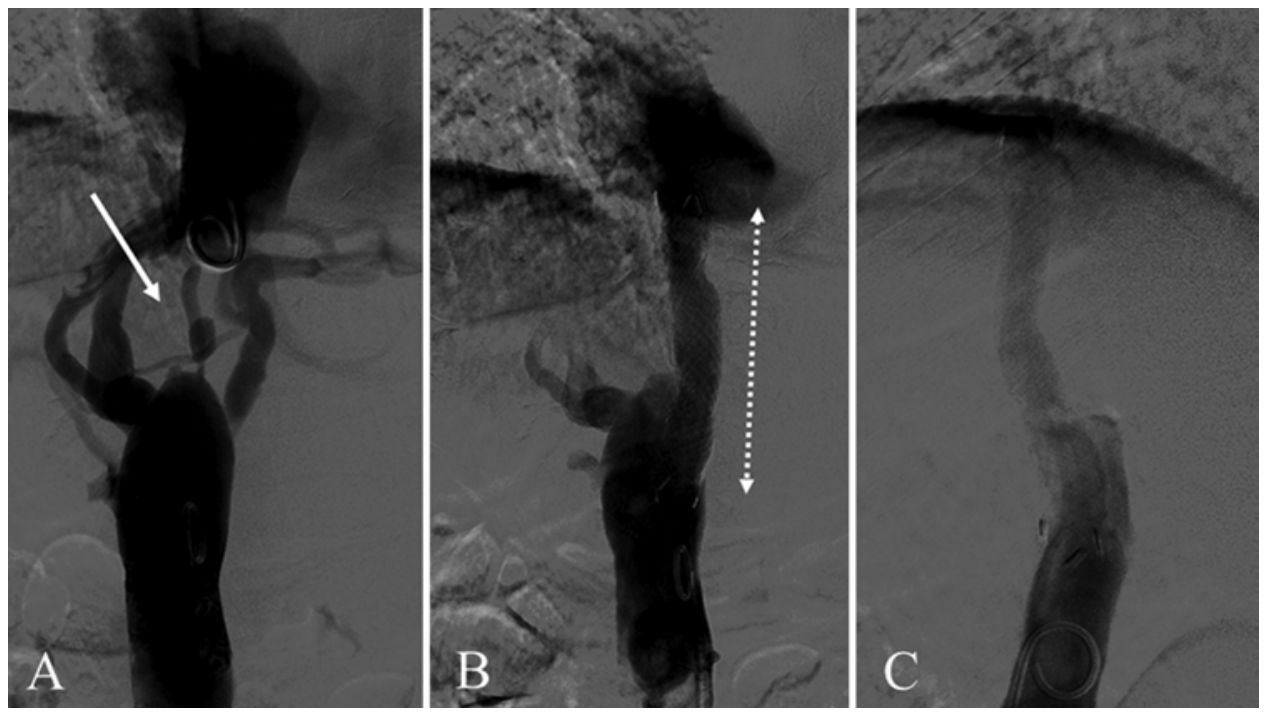

FIG. 2. A: Intrahepatic IVC recanalization was performed. IVC venogram confirmed a 3-cm-long total occlusion (white arrow). B: Puncture was done with a 16-gauge Colapinto needle and a 12-mm/8-cm stent graft was inserted after preballooning with an IN.PACT Admiral drug-eluting balloon (dotted line). C: Tachogram after postballooning confirmed the shift of main blood flow to the stent graft, accompanied by decreased perfusion via collateral vessels.

angiography revealed that the patency of the stent graft was maintained in the intrahepatic IVC. Furthermore, the degree of dilation of collateral veins was less than that prior to intrahepatic IVC recanalization. At the 3-month follow-up, the patient showed marked improvement of lower-extremity weakness (motor power grade: hip flexion $5 / 5$, knee extension $5 / 5$, ankle dorsiflexion $5 / 5$, ankle plantar flexion $5 / 5$, big toe dorsiflexion $5 / 3$, and big toe plantar flexion 5/4). Moreover, the patient reported improvement in voiding difficulty and decreased pain in his back and both lower extremities.

At the 1-year follow-up, the patient had no recurrent episodes of pain or numbness of either lower extremity. The patient complained of nonspecific intermittent lower-ex-
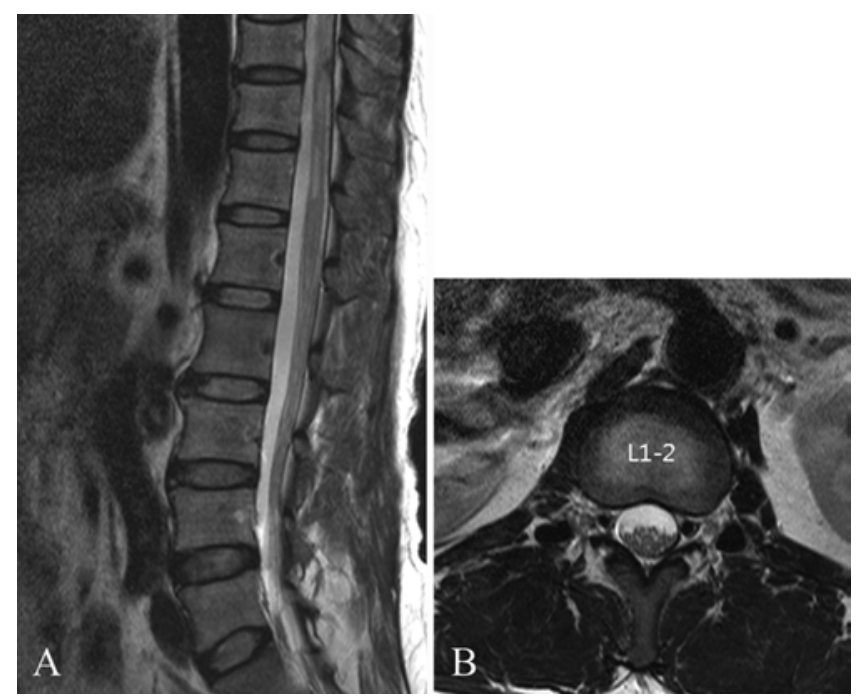

FIG. 3. A and B: At the 1-month follow-up, thoracolumbar MRI scans showed a marked decrease in epidural venous engorgement but also revealed persistent syringomyelia at the lower thoracic levels. tremity weakness, but his motor power grade results were slightly improved compared with the 3 -month postoperative follow-up, and he reported that his activities of daily living were not disrupted by the mild motor weakness.

\section{Discussion}

Budd-Chiari syndrome is rare, with an estimated prevalence of 1/1,000,000 individuals. Its etiologies include venous obstruction or occlusion of the hepatic veins and/or a retrohepatic IVC. Patients with Budd-Chiari syndrome present with clinical findings such as portal and IVC hypertension..$^{9}$ In these patients, collateral vein engorgement due to IVC occlusion may extend to the epidural or interforaminal space; this may eventually lead to symptoms that are suggestive of disc herniation or foraminal stenosis. ${ }^{10}$ Bozkurt et al. ${ }^{1}$ reported a case of radicular and low-back pain secondary to IVC stenosis in a patient with Budd-Chiari syndrome that was successfully treated with an endovascular procedure. To our knowledge, ours is the second reported case involving epidural venous engorgement due to Budd-Chiari syndrome Type I, which has occlusion of hepatic veins by thrombosis, not compression of an outside structure. Of note, our patient presented with more severe symptoms than the patient described in the previously reported case.

In the current case, the patient presented with epidural venous engorgement and syringomyelia arising from IVC obstruction due to Budd-Chiari syndrome, with symptoms suggestive of myelopathy, including lower-extremity weakness and voiding difficulty. In most previously reported cases, patients presented with radiating pain or neurogenic claudication arising from epidural varices. ${ }^{1,2,3,5,10}$ In the current case, however, the patient complained of more serious complications indicative of myelopathy. It is usually considered mandatory to perform surgical exploration in patients presenting with symptoms indicative of myelopathy, including lower-extremity weakness, gait disturbance, 
and bladder dysfunction. However, our patient presented with symptoms that had occurred for a long period of time without abrupt neurological progression and he had a definite cause of epidural engorgement. We therefore decided to perform radiological interventions to treat IVC stenosis before spine surgery. At the 3-month follow-up, the patient showed great improvement in lower-extremity weakness.

There are some reports of epidural venous enlargement accompanied by radicular and/or back pain., 3,6,11 Paksoy and Gormus ${ }^{10}$ reported 13 cases of epidural varices accompanied by radicular symptoms that were indicative of lumbar disc herniation or spinal stenosis, and on the basis of these cases the authors suggested that epidural venous engorgement might cause radicular and/or back pain in patients with deep venous and IVC thrombosis. Genevay et al. ${ }^{5}$ reported 2 cases of lumboradiculopathy secondary to nonthrombosed epidural varices, for which these authors performed surgical treatment. Moreover, in both of these cases the patients showed symptoms of cervical myelopathy due to epidural venous engorgement arising from shunt-related intracranial complications. ${ }^{7,12}$

To our knowledge, there are no previous reports of patients with thoracolumbar myelopathy due to epidural venous engorgement. In the current case, the patient complained of symptoms indicative of thoracolumbar myelopathy, including lower-extremity weakness, gait disturbance, and voiding difficulty. The patient had severe venous engorgement due to IVC obstruction caused by Budd-Chiari syndrome and syringomyelia at the lower thoracic levels. Furthermore, the patient had the neurological deficits of lower-extremity weakness and voiding difficulty. We therefore assumed that the syringomyelia and symptoms indicative of myelopathy arose from severe mechanical compression at the thoracolumbar levels, which decreased the dural blood supply. However, even in a patient with such serious symptoms, elimination of the predisposing causes by interventional stent insertion led to shrinkage of the epidural venous engorgement as well as correction of the patient's serious neurological deficits without the need for surgical exploration. Other options for intrahepatic IVC recanalization include thrombolysis and surgical shunts. However, direct infusion of thrombolytic agents is not routinely attempted, and shunts should be placed early after diagnosis and have relatively higher procedure-related mortality than the use of a stent.

This case indicates that the spinal cord or dural sac compression from venous engorgement may elicit symptoms that are suggestive of myelopathy or radiculopathy in patients with Budd-Chiari syndrome. 3D CT angiography is a useful modality to establish a diagnosis of Budd-Chiari syndrome and to accurately identify lesions. In addition, IVC recanalization using an interventional radiology technique, such as insertion of a stent graft, is a useful modality to restore the perfusion. Decreased venous engorgement can be evaluated on follow-up imaging studies, and in our case, the patient's functional deterioration and pain at baseline improved and returned to normal status without spinal surgery.

In conclusion, this case highlights the possibility that epidural venous engorgement at the thoracolumbar level may cause symptoms that are suggestive of myelopathy, such as lower-extremity weakness and voiding difficulty. Furthermore, our case demonstrates that these symptoms can be alleviated by treatment that eliminates the underlying causes of spinal canal encroachment.

\section{References}

1. Bozkurt G, Cil B, Akbay A, Türk CC, Palaoğlu S: Intractable radicular and low back pain secondary inferior vena cava stenosis associated with Budd-Chiari syndrome: endovascular treatment with cava stenting: case report and review of the literature. Spine (Phila Pa 1976) 31:E383-E386, 2006

2. Dabasia H, Rahim N, Marshall R: Neurogenic claudication without spinal stenosis arising as a result of lumbar epidural varices. J Bone Joint Surg Br 94:1292-1294, 2012

3. Demaerel P, Petré C, Wilms G, Plets C: Sciatica caused by a dilated epidural vein: MR findings. Eur Radiol 9:113-114, 1999

4. Geibprasert S, Pongpech S, Jiarakongmun P, Krings T: Cervical spine dural arteriovenous fistula presenting with congestive myelopathy of the conus. J Neurosurg Spine 11:427-431, 2009

5. Genevay S, Palazzo E, Huten D, Fossati P, Meyer O: Lumboradiculopathy due to epidural varices: two case reports and a review of the literature. Joint Bone Spine 69:214-217, 2002

6. Hammer A, Knight I, Agarwal A: Localized venous plexi in the spine simulating prolapse of an intervertebral disc: a report of six cases. Spine (Phila Pa 1976) 28:E5-E12, 2003

7. Liu JK, Gottfried ON, Brockmeyer DL: Epidural venous engorgement resulting in progressive cervical myelopathy from shunt-related intracranial hypotension. Case report and review of the literature. J Neurosurg 105 (6 Suppl):499-503, 2006

8. Mohit AA, Fisher DJ, Matthews DC, Hoffer E, Avellino AM: Inferior vena cava thrombosis causing acute cauda equina syndrome. Case report. J Neurosurg 104 (1 Suppl):46-49, 2006

9. Okuda K, Kage M, Shrestha SM: Proposal of a new nomenclature for Budd-Chiari syndrome: hepatic vein thrombosis versus thrombosis of the inferior vena cava at its hepatic portion. Hepatology 28:1191-1198, 1998

10. Paksoy Y, Gormus N: Epidural venous plexus enlargements presenting with radiculopathy and back pain in patients with inferior vena cava obstruction or occlusion. Spine (Phila Pa 1976) 29:2419-2424, 2004

11. Pennekamp PH, Gemünd M, Kraft CN, von Engelhardt LV, Lüring C, Schmitz A: [Epidural varicosis as a rare cause of acute radiculopathy with complete foot paresis-case report and literature review.] Z Orthop Ihre Grenzgeb 145:55-60, 2007 (Ger)

12. Ulrich NH, Maier M, Bernays RL, Krayenbuhl N, Kollias S: Cervical myelopathy due to chronic overshunting in a pediatric patient: case report and review of the literature. Turk Neurosurg 23:410-414, 2013

\section{Author Contributions}

Conception and design: Lee. Acquisition of data: Lee, Song. Analysis and interpretation of data: Kang. Drafting the article: Lee, Song. Critically revising the article: Kang, Lee. Reviewed submitted version of manuscript: Song. Approved the final version of the manuscript on behalf of all authors: Kang. Administrative/technical/material support: Lee, Song.

\section{Correspondence}

Kyung-Chung Kang, Department of Orthopedic Surgery, Kyung Hee Medical Center, Kyung Hee University School of Medicine, Seoul 130-702, Republic of Korea. email: futurespine@gmail. com. 